\title{
Giant Dipole Resonance Width in near-Sn Nuclei at Low Temperature and High Angular Momentum
}

\author{
Srijit Bhattacharya, ${ }^{1}$ S. Mukhopadhyay, ${ }^{2}$ Deepak Pandit, ${ }^{2}$ Surajit Pal, ${ }^{2}$ A. \\ De ${ }^{3}$ S. Bhattacharya, ${ }^{2}$ C. Bhattacharya, ${ }^{2}$ K. Banerjee, ${ }^{2}$ S. Kundu, ${ }^{2}$ T.K. \\ Rana, ${ }^{2}$ A. Dey, ${ }^{2}$ G. Mukherjee, ${ }^{2}$ T. Ghosh,${ }^{2}$ D. Gupta,${ }^{2}$ and S. R. Banerjee ${ }^{2} * *$ \\ ${ }^{1}$ Darjeeling Government College, Darjeeling-734 101, India \\ ${ }^{2}$ Variable Energy Cyclotron Centre, \\ 1/AF Bidhan Nagar, Kolkata-700 064, India \\ ${ }^{3}$ Raniganj Girls' College, Raniganj-713 347, India
}

(Dated: August 30, 2021)

\begin{abstract}
High energy $\gamma$-rays in coincidence with low energy yrast $\gamma$-rays have been measured from ${ }^{113} \mathrm{Sb}$, at excitation energies of 109 and $122 \mathrm{MeV}$, formed by bombarding ${ }^{20} \mathrm{Ne}$ on ${ }^{93} \mathrm{Nb}$ at projectile energies of 145 and $160 \mathrm{MeV}$ respectively to study the role of angular momentum (J) and temperature (T) over Giant Dipole Resonance (GDR) width $(\Gamma)$. The maximum populated angular momenta for fusion were $67 \hbar$ and $73 \hbar$ respectively for the above-mentioned beam energies. The high energy photons were detected using a Large Area Modular $\mathrm{BaF}_{2}$ Detector Array (LAMBDA) along with a 24-element multiplicity filter. After pre-equilibrium corrections, the excitation energy $\mathrm{E}^{*}$ was averaged over the decay steps of the compound nucleus $(\mathrm{CN})$. The average values of temperature, angular momentum, CN mass etc. have been calculated by the statistical model code CASCADE. Using those average values, results show the systematic increase of GDR width with $\mathrm{T}$ which is consistent with Kusnezov parametrization and the Thermal Shape Fluctuation Model. The rise of GDR width with temperature also supports the assumptions of adiabatic coupling in the Thermal Shape Fluctuation Model. But the GDR widths and corresponding reduced plots with J are not consistent with the theoretical model at high spins.
\end{abstract}

PACS numbers: 24.30.Cz, 24.60.Dr, 25.70.Gh, 27.60.+j 


\section{INTRODUCTION}

The study of nuclear structure and dynamics under extreme conditions of internal energy and angular momentum is important in understanding the diverse properties of atomic nuclei. The measurements of high energy gamma rays emitted when isovector Giant Dipole Resonances (GDR) in highly excited nuclei are damped can provide information on the various nuclear properties at finite temperature (T) and angular momentum (J) [1, 2]. Although the excited state GDR in heavy ion fusion reactions was observed in the early eighties, the study of this resonance still continues to be a very interesting and useful tool in the field of nuclear structure and dynamics [3]. The systematics of GDR width $(\Gamma)$ as a function of $\mathrm{T}$ and $\mathrm{J}$ still remains a hotly debated and puzzling topic. The central issue is to understand the role of different damping mechanisms viz. collisional damping [4] and adiabatic thermal shape fluctuation [5, 6] with their dependence on $\mathrm{T}$ and rotational frequency of the nucleus.

Till now most of the measurements of GDR cross-section built on excited states have been made with Sn and near-Sn nuclei formed by heavy ion fusion reactions. Previous measurements [1, 7, 8, 9, 10] suggest the continuous growth of GDR width with excitation energy $\left(E^{*}\right)$ up to $120-130 \mathrm{MeV}(\mathrm{T} \leq 2 \mathrm{MeV})$ and attribute the same to rapid increase of spin-induced deformations and thermal shape fluctuations. As per the experimental observations, beyond the bombarding energy at which angular momentum saturates, the increase in GDR width is very small. This saturation of width is interpreted as the evidence for the onset of maximum angular momentum the nucleus can sustain without fissioning. Theoretically, the Thermal Shape Fluctuation Model (TSFM), in general, can predict the trend of the experimental data for $E^{*} \leq 120-130 \mathrm{MeV}$ but after that it fails to show any saturation of GDR width with increasing $\mathrm{E}^{*}$. On the contrary, the effect of temperature on GDR width, as is revealed from the work of Kelly et al. [11] is quite inconsistent with the saturation previously observed. Kelly et al. emphasized that at higher bombarding energies the excitation energy and temperature should be correctly redefined. Their work results in a very interesting observation that if nuclear temperature is estimated using average $\mathrm{E}^{*}$ after proper pre-equilibrium corrections, previous results could also indicate the increase of GDR width up to $\mathrm{T} \leq 3.5 \mathrm{MeV}$ (unlike up to $2 \mathrm{MeV}$ as previoulsy observed). The general trend of those experimental data agrees well with the predictions of the TSFM. In spite of that, some recent observations, in the region $\mathrm{T} \leq 2 \mathrm{MeV}$, show that the experimental findings of 
GDR width are smaller than the predictions of TSFM in at least 4 different mass regions $(\mathrm{Cu}, \mathrm{Sn}, \mathrm{Pb}, \mathrm{Au})[3,12,13,14]$.

Very recently, the study of angular momentum dependence of $\Gamma$ keeping $E^{*}$ unchanged has become more controversial. Previous experimental findings agree with the fact that $\Gamma$ remains constant up to $\mathrm{J} \sim 30-40 \hbar$ which is in conformity with Kusnezov parametrization of TSFM. However, at higher J values a few recent results seem to violate this parametrization. In the region $\mathrm{T} \leq 2 \mathrm{MeV}$ and with higher angular momenta, the TSFM can explain the variation of $\Gamma$ in the case of ${ }^{106} \mathrm{Sn}$ and ${ }^{176} \mathrm{~W}$ [15, 16] but fails for ${ }^{86} \mathrm{Mo}$ to do so [17]. The recent investigation by Chakrabarty [18] emphasizes that the average values of temperature, angular momentum and mass must be smaller than those of the initial compound nucleus considered. Interestingly, if the average values of those parameters are taken into account, Kusnezov parametrization can successfully explain the experimental data of ${ }^{86}$ Mo but it fails

in the case of Sn [18]. Thus though the model of thermal shape fluctuation describes rather well, on the average, many experimental results, it fails to reproduce the data corresponding to the lowest temperatures and highest spins.

Under these circumstances, it comes out to be that plenty of experimental data are needed in the region of $\mathrm{T} \leq 2 \mathrm{MeV}$ with higher angular momenta to understand the limits of the TSFM and Kusnezov parametrization.

The present experiment revisits excited state GDR in ${ }^{113} \mathrm{Sb}$ - a near $\mathrm{Sn}$ nuclide, to provide new results at higher spins (in the region of 40-60 $\hbar$ ) in order to test the simple parametrization given by Kusnezov, which is based on, mainly, the data at low and medium spins. The values of $\mathrm{E}^{*}, \mathrm{~J}$ and mass $(\mathrm{A})$ have been estimated by averaging over the decay steps of the compound nucleus. In this work an effort is made to provide the temperature of the emitting nucleus applying all the necessary corrections including that of the pre-equilibrium emission. The pre-equilibrium corrections have been done using the parametrization of Kelly et al. [11].

\section{EXPERIMENTAL DETAILS}

The experiment was performed at the Variable Energy Cyclotron Centre (VECC), Kolkata. A $1 \mathrm{mg} / \mathrm{cm}^{2}$ thick target of $99.9 \%$ pure ${ }^{93} \mathrm{Nb}$ was bombarded with a beam of ${ }^{20} \mathrm{Ne}$ produced by the K130 Cyclotron of VECC. Two different beam energies of $145 \mathrm{MeV}$ 


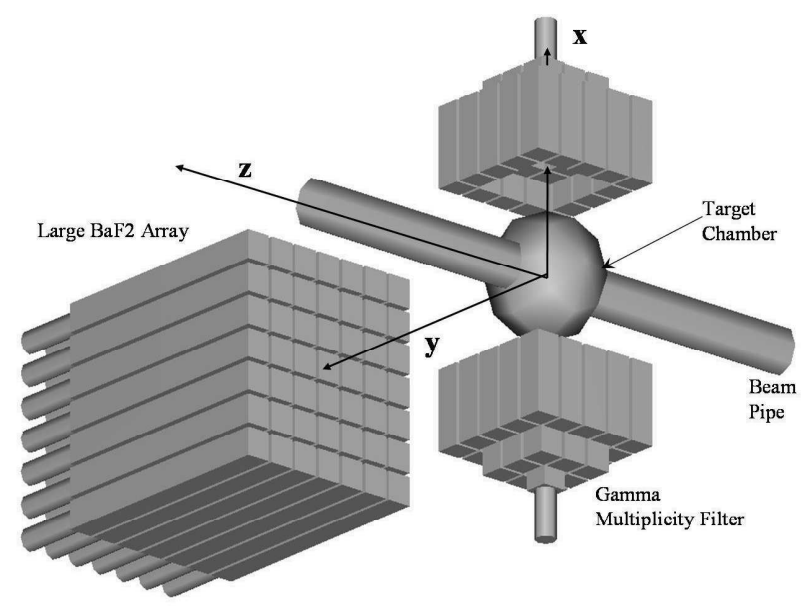

FIG. 1: Schematic view of experimental set-up for the LAMBDA (Large $\mathrm{BaF}_{2}$ array) spectrometer in a $7 x 7$ matrix arrangement along with the low energy $\gamma$-ray multiplicity filter.

and $160 \mathrm{MeV}$ were employed forming the compound nucleus ${ }^{113} \mathrm{Sb}$ at the excitation energies of $109 \mathrm{MeV}$ and $122 \mathrm{MeV}$ respectively. The maximum populated angular momenta for fusion were $67 \hbar$ and $73 \hbar$ respectively at the two bombarding energies. The inclusive high energy $\gamma$-rays were detected with a part of the $\mathrm{BaF}_{2}$ detector array LAMBDA [19, 20]. The array comprised of 49 detectors arranged in a $7 \times 7$ square matrix configuration, each detector having a length of $35 \mathrm{~cm}$ and a square face of $3.5 \times 3.5 \mathrm{~cm}^{2}$ area. The detector array was positioned at a distance of $50 \mathrm{~cm}$ from the target and at an angle of $55^{\circ}$ w.r.t. the beam axis. The array subtended a solid angle of $0.227 \mathrm{sr}(1.8 \%$ of $4 \pi)$. Lead sheets of 3 $\mathrm{mm}$ thickness were placed in front and sides of the array to cut down the low energy $\gamma$-rays and X-rays. The beam dump was heavily shielded with borated paraffin and lead bricks to decrease the neutron and $\gamma$-ray background. A 24-element multiplicity filter detector array was used along with the LAMBDA spectrometer to measure the multiplicity of low energy $\gamma$-rays in coincidence with the high energy $\gamma$-rays. The multiplicity detector assembly also consists of $\mathrm{BaF}_{2}$ detectors, each $3.5 \times 3.5 \times 5.0 \mathrm{~cm}^{3}$ in dimension, packed in two groups of 12 each and placed on the two sides of the target chamber at a distance of $10 \mathrm{~cm}$ from the target. The complete detection system is shown schematically in the Fig. 1,

The response of the LAMBDA spectrometer [20] was generated using the Monte Carlo Code GEANT 3.21 [21] incorporating realistic geometry of the array, the energy resolution of the detectors, experimental conditions of shielding, discriminator thresholds etc. The energy calibration of the individual detectors was done using the low energy $\gamma$-ray sources 


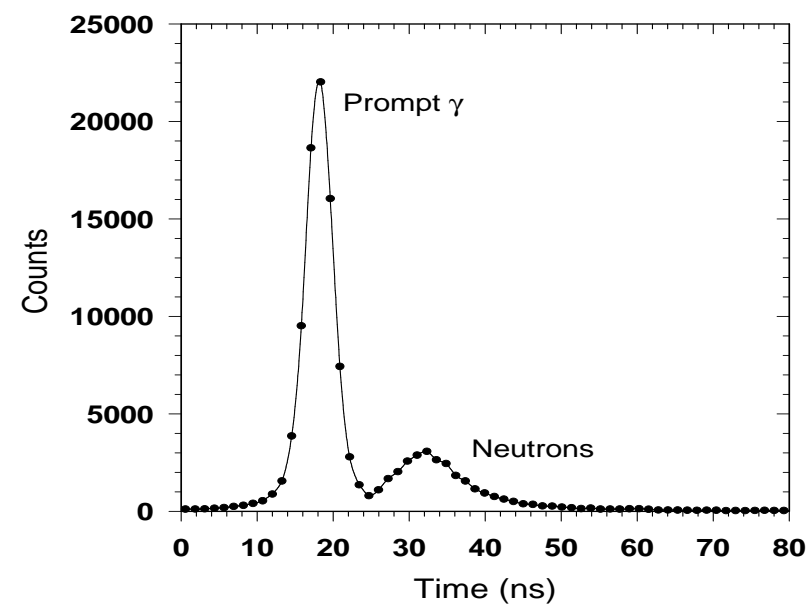

FIG. 2: The experimental time spectrum obtained from a single detector showing a clear separation between the neutrons and prompt $\gamma$-rays.

viz. ${ }^{22} \mathrm{Na}(0.511 \mathrm{MeV}, 1.274 \mathrm{MeV}$, and the sum peak $1.785 \mathrm{MeV}),{ }^{60} \mathrm{Co}$ (Sum Peak 2.505 $\mathrm{MeV}),{ }^{241} \mathrm{Am}-{ }^{9} \mathrm{Be}(4.43 \mathrm{MeV})$ and also by minimum ionising peak (23.1 MeV) of cosmic muons. The energy response of the detectors was found to be linear up to $23.1 \mathrm{MeV}$. The time resolution of individual detectors was 960 ps. The response of the $\gamma$-ray multiplicity detector array was also generated using GEANT 3.21 simulation code [22]. A dedicated electronics setup (consisting of multi-inputs CAMAC and NIM modules and a VME based data acquisition system capable of handling $\sim 4 \mathrm{~K}$ events/second without appreciable deadtime loss) was used to register the energy and time from each detector in an event by event mode. An event was treated as a valid event only when the deposited energy in any detector crosses a high threshold $\left(\mathrm{T}_{h}\right)$ of $4 \mathrm{MeV}$. The details of high energy gamma spectrometer LAMBDA, its response, electronics setup and event reconstruction method have already been described in S.Mukhopadhyay et al. [20].

Time of Flight (TOF) technique was used to eliminate neutrons. For TOF measurements, the time reference was taken from the multiplicity detector assembly. A clear separation between the neutrons and prompt $\gamma$-rays was seen for all the detector elements in the experimental time spectra (Fig. 2). Pulse Shape Discrimination (PSD) method by long $(2 \mu \mathrm{s})$ - short (50 ns) integration technique was adopted to reject pile-up events. Since the detector array is highly segmented, pile-up events were very small. In triggered data acquisition mode the probability of cosmic events is small (rejection ratio better than 1:3300) and also those events can be rejected effectively from the hit pattern utilizing the square 
detector geometry and high segmentation of the array.

\section{EXPERIMENTAL DATA ANALYSIS}

The high energy $\gamma$-ray spectra were generated from the event-by-event data during offline analysis. For reconstructing the events, a nearest neighbor (cluster) summing technique was adopted. In this technique, first, the detector with highest energy deposited above a high threshold $(\geq 4 \mathrm{MeV})$ within the array was identified and named as primary detector. For

obtaining the full energy information of the incident photons, it is important to confine the secondary electromagnetic shower within the array volume as far as possible. Therefore, in the above-mentioned event reconstruction technique a checking was done to find whether the primary detector was surrounded by all its neighbors i.e., all the 8 elements (irrespective of any hits in them). The event was treated as valid, if this condition was satisfied. Otherwise, due to the possibility of losing a part of the electromagnetic shower, the event was rejected. In the case of a valid event, for the final adding back, only those detectors were considered among the 8 nearest neighbors, having an energy deposit $>250 \mathrm{keV}$. The same scheme had been adopted while simulating the response of the array using GEANT. During the adding back, the hit events in the individual elements in the cluster were validated by the prompt gamma cuts in TOF spectra and long-short PSD selections. Next by gating on different coincidence folds of low energy $\gamma$-multiplicities in the multiplicity array the high energy $\gamma$-ray spectra were generated for each beam energy. The contributions due to the chance coincidence events within the prompt $\gamma$ window in the TOF spectrum were also subtracted. Finally the spectra were Doppler corrected.

The conversion between the measured coincidence fold $F_{\gamma}$ (the number of measured coincident $\gamma$-rays of low energy in each event) to the multiplicity $M_{\gamma}$ (the number of $\gamma$-rays emitted in the reaction) was established using the response matrix $S\left(F_{\gamma}, M_{\gamma}\right)$. This response matrix was generated by making use of the GEANT, where, realistic multiplicity detector setup was considered and low energy $\gamma$-rays were thrown isotropically with incident multiplicity distribution $\mathrm{P}(\mathrm{M})$. It has been assumed that the multiplicity distribution following fusion reaction is given by [16],

$$
\mathrm{P}(\mathrm{M})=\mathrm{M}_{\gamma} /\left[1+\exp \left\{\left(M_{\gamma}-M_{0}\right) / \delta M\right\}\right]
$$

The maximum of multiplicity $\mathrm{M}_{0}$ and diffuseness $\delta \mathrm{M}$ were obtained by fitting the equa- 


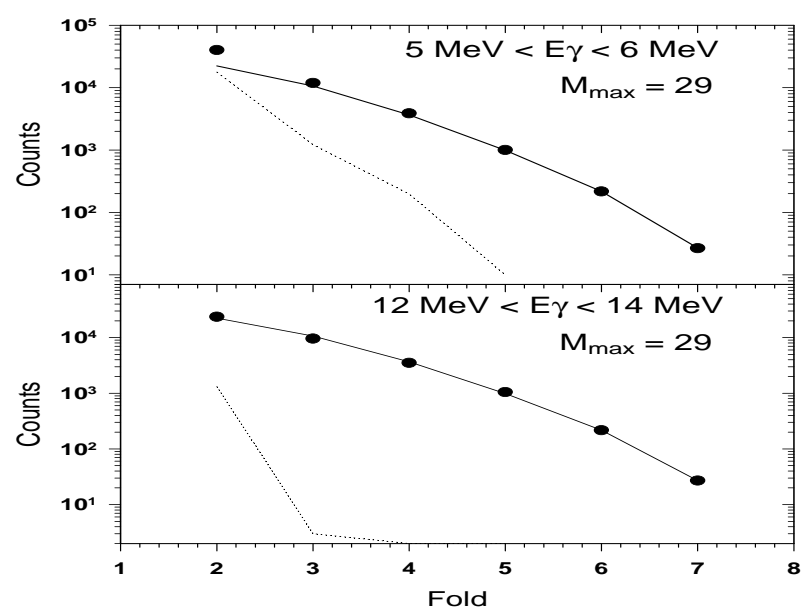

FIG. 3: Experimental fold spectrum fitted with GEANT simulation (solid line) for the high energy $\gamma$ windows $5 \mathrm{MeV}<E_{\gamma}<6 \mathrm{MeV}$ (top panel) and for $12 \mathrm{MeV}<E_{\gamma}<14 \mathrm{MeV}$ (bottom panel) for beam energy $=160 \mathrm{MeV}$. The dotted line is the difference between the measured and predicted fold distributions.

tion,

$$
\sum S\left(F_{\gamma}, M_{\gamma}\right) P\left(M_{\gamma}\right)=f_{\exp }\left(F_{\gamma}\right)
$$

where $\mathrm{f}_{\text {exp }}\left(E_{\gamma}\right)$ is the measured multiplicity spectrum in the region $2 \leq \mathrm{F}_{\gamma} \leq 7$. Typical experimental fold distributions are shown in the Fig. 3.

The theoretical fold distributions were found to be matching well with the experimental fold distributions for 145 and $160 \mathrm{MeV}$ for $E_{\gamma} \geq 12 \mathrm{MeV}$. For $4 \mathrm{MeV} \leq \mathrm{E}_{\gamma}<12 \mathrm{MeV}$, another low multiplicity component had to be added. This actually is the difference between experimentally measured and theoretically predicted fold distributions and is shown by the dotted line in the Fig. 3. The intensity of the low multiplicity component peaks at around $7 \mathrm{MeV}$ and falls on either side becoming negligible beyond $12 \mathrm{MeV}$. Its contribution was estimated for each fold from the fits of the fold distributions. The correction factors for the high energy $\gamma$-ray spectra were generated from the percentage contribution of the abovementioned low multiplicity component. The correction factors are plotted in the Fig. 4 against the $\gamma$-ray energies corresponding to different folds for $160 \mathrm{MeV}$ incident energy. The corrections decreased as a function of fold and became negligibly small for fold $\mathrm{F} \geq 4$. The high energy $\gamma$-ray spectra corresponding to selected folds were multiplied (in the region below $12 \mathrm{MeV}$ ) by the energy dependent correction factors and corrected accordingly. The Fig. 5 
TABLE I: Table showing the fitted values of parameters of multiplicity and angular momentum distributions.

\begin{tabular}{|c|r|r|r|}
\hline \hline $\mathrm{E}_{\text {proj }}$ & $\mathrm{M}_{\max }$ & $\delta \mathrm{M}$ & $\mathrm{J}_{\max }$ \\
$(\mathrm{MeV})$ & & & $(\hbar)$ \\
\hline 145 & 28 & 3 & 60 \\
160 & 29 & 3 & 62 \\
\hline \hline
\end{tabular}

shows a typical high energy $\gamma$-ray spectrum (for fold $=2$ at $160 \mathrm{MeV}$ projectile energy) thus corrected (filled circles) along with the raw uncorrected one (open circles). The measured enhanced yield at low folds could be due to non-fusion events. Since the reaction studied has an asymmetry between $\mathrm{N} / \mathrm{Z}$ of the target and projectile, there might be a possibility of pre-equilibrium $\gamma$-ray emissions as those are related to the dynamic dipole formation in the fusion entrance channel. However, since the recoiling nucleus was not directly measured it is not possible to disentangle the different effects.

From the multiplicity distributions for different folds, the angular momentum $(\mathrm{J})$ distributions of the compound nucleus have been extracted and the average value of $\mathrm{J}\left(\mathrm{J}_{C N}\right)$ for the compound nucleus was calculated. The conversion from multiplicity to angular momentum was done assuming $\mathrm{J}=2 \mathrm{M}+\mathrm{k}, \mathrm{k}=4$ takes into account the angular momentum removed by nonstatistical gamma rays, particle emission and gamma rays below trigger thresholds. The fitted values of parameters $\delta \mathrm{M}, M_{\max }, J_{\max }$ with corresponding beam energies are shown in Table I. The average angular momenta and the corresponding widths for different folds are shown in Table II.

\section{Statistical Model Analysis}

The high energy $\gamma$-ray data collected in the experiment were sorted into 4 different classes corresponding to folds $2,3,4$ and $\geq 5$. The measured high energy $\gamma$ spectra associated with different folds were fitted with a modified version of statistical model code CASCADE [23] along with a bremsstrahlung component. There is enough experimental evidence that at projectile energy above $6 \mathrm{MeV} / \mathrm{u}$, contribution of pre-equilibrium particle emission becomes important and should be included in CASCADE. For calculation of proper $\mathrm{E}^{*}$, pre-equilibrium 


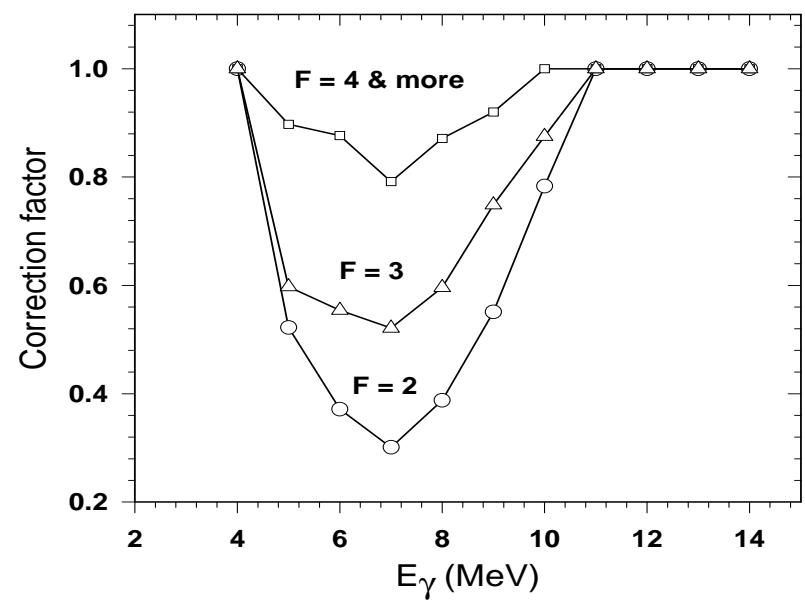

FIG. 4: The correction factors are plotted against $\gamma$-ray energies for fold 2 (circles), 3 (triangles) and $\geq 4$ (squares) in the case of $\mathrm{E}_{\text {beam }}=160 \mathrm{MeV}$. The decrease of correction factors (see text for details) with the increase of fold is evident.

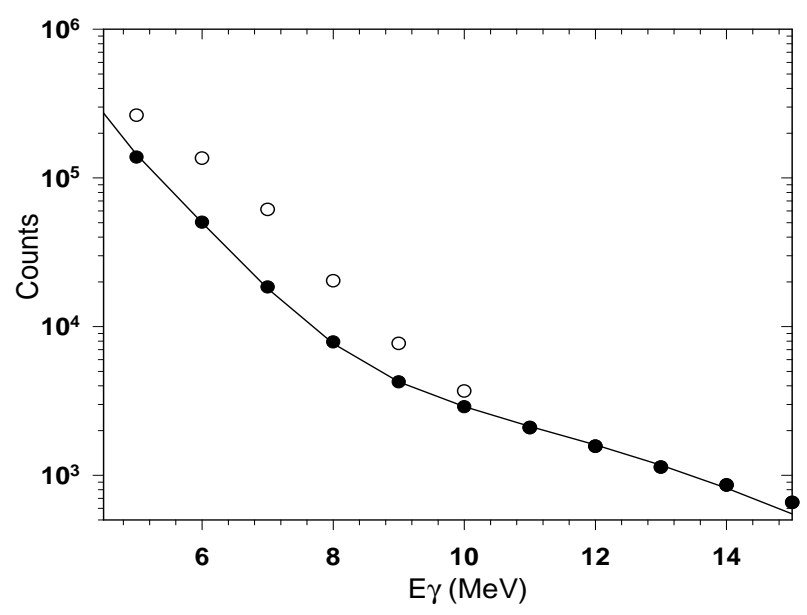

FIG. 5: High energy $\gamma$-ray spectra (expanded up to $15 \mathrm{MeV}$ ) corresponding to fold 2 at 160 $\mathrm{MeV}$ beam energy before (shown by open circles) and after corrections (shown by filled circles). Corresponding statistical model fit is shown by solid lines. The error bars of the respective points are less than the size of the symbols. 
TABLE II: Table showing the angular momenta and corresponding widths for different folds at two beam energies.

\begin{tabular}{|c|r|r|r|}
\hline \hline $\mathrm{E}_{\text {proj }}$ & fold & $\mathrm{J}_{C N}$ & FWHM in J \\
$(\mathrm{MeV})$ & $(\hbar)$ & $(\hbar)$ & $(\hbar)$ \\
\hline 145 & 2 & 49 & 24 \\
145 & 3 & 53 & 22 \\
145 & 4 & 57 & 18 \\
145 & $\geq 5$ & 59 & 16 \\
160 & 2 & 50 & 24 \\
160 & 3 & 54 & 20 \\
160 & $\geq 4$ & 59 & 18 \\
\hline \hline
\end{tabular}

estimates were done on the basis of the empirical formula [11]

$$
\Delta E_{x}(\mathrm{MeV})=8.7\left[\left(E_{\text {proj }}-V_{c}\right) / A_{\text {proj }}\right]-33
$$

where $V_{c}$ is the Coulomb barrier. This parametrization based on the demonstrated scaling with $\left(E_{\text {proj }}-V_{c}\right) / A_{\text {proj }}$ (insensitive to the target-projectile combinations [24]) have been used in this work to estimate the energy lost in pre-equilibrium emission and to correct the excitation energy $\left(V_{c}=57.0 \mathrm{MeV}\right.$ at $\left.\mathrm{r}=\mathrm{r}_{c}=10.35 \mathrm{fm}\right)$. Corresponding pre-equilibrium energy loss is $5.28 \mathrm{MeV}$ at $E_{l a b}=145 \mathrm{MeV}$ ( $5 \%$ of initial excitation energy) and $11.8 \mathrm{MeV}$ at $E_{l a b}=160 \mathrm{MeV}$ (10\% of initial excitation energy). The corrected excitation energy was used within CASCADE, in which Reisdorf level density prescription [25] had been used. The asymptotic level density parameter [26] was taken as $\tilde{a}=\mathrm{A} / 8.0 \mathrm{MeV}^{-1}$. In the statistical model calculation, a single lorentzian GDR strength function was assumed, having centroid energy $\left(\mathrm{E}_{G D R}\right)$, strength $(\mathrm{S})$, width $(\Gamma)$ as parameters . In the CASCADE calculation, the moment of inertia I of the compound nucleus was taken as, $\mathcal{I}=\mathcal{I}_{0}\left(1+\delta_{1} J^{2}+\delta_{2} J^{4}\right)$ where $\mathcal{I}_{0}$ is the spherical moment of inertia. The parameters $r_{e f f}, \delta_{1}$ and $\delta_{2}$ were kept at default values of $1.22 \mathrm{fm}, 0.4699 \times 10^{-5}$ and $0.9326 \times 10^{-8}$ respectively within the CASCADE. The spin distributions for different folds of the compound nucleus deduced from the experimental multiplicity distribution were used as inputs in CASCADE. The critical angular momenta $l_{c r}$, fusion cross sections and the corresponding projectile energies are shown in Table III. 
TABLE III: Table showing critical angular momenta, fusion cross-sections for each beam energy calculated by CASCADE.

\begin{tabular}{llc}
\hline \hline $\mathrm{E}_{\text {beam }}$ & $\mathrm{J}_{\text {cr }}$ & $\sigma_{\text {fus }}$ \\
$(\mathrm{MeV})$ & $(\hbar)$ & $(\mathrm{mb})$ \\
\hline 145 & 67 & 1390 \\
160 & 73 & 1450 \\
\hline \hline
\end{tabular}
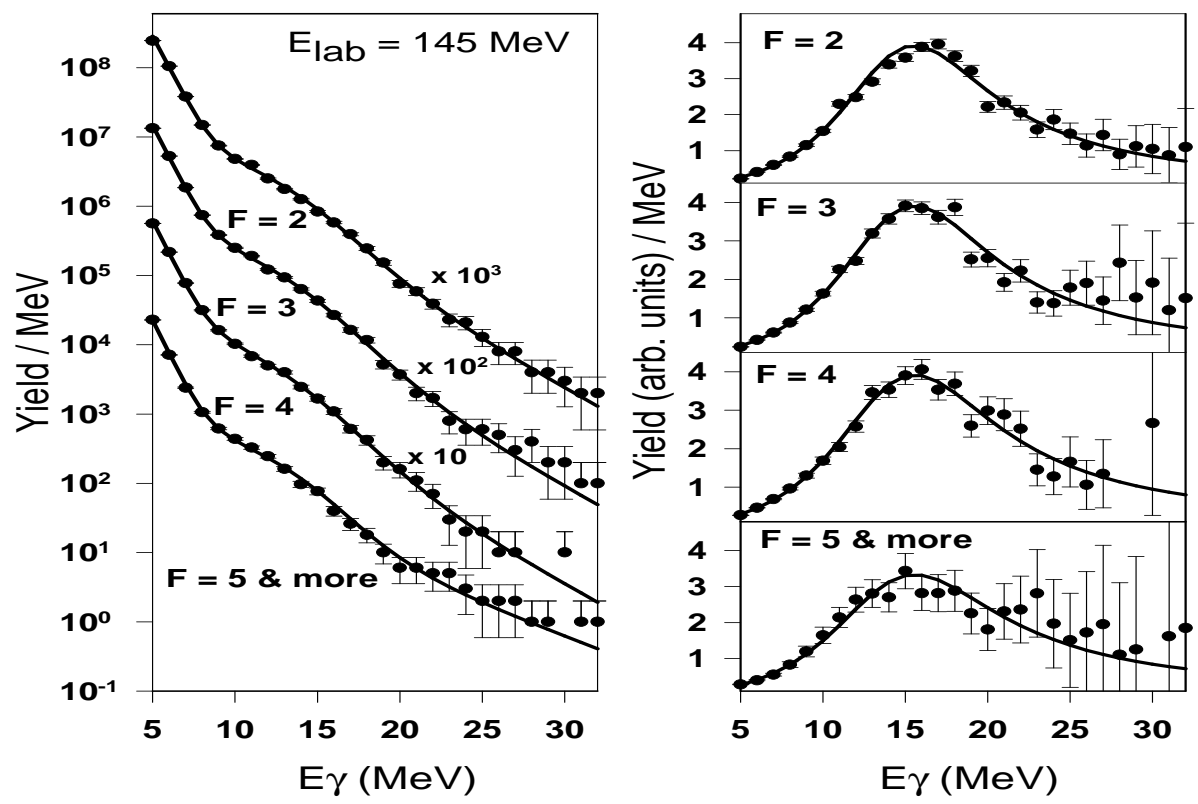

FIG. 6: Left: high energy $\gamma$-ray spectra for different folds for beam energy $145 \mathrm{MeV}$ are plotted, right: the linearized GDR spectra for different folds are plotted with $\gamma$-ray energies.

\section{Bremsstrahlung Contribution}

The non-statistical contributions to the experimental $\gamma$-ray spectra arising from bremsstrahlung processes were assumed to have an energy dependence of $\exp \left(-E_{\gamma} / E_{0}\right)$, where the slope parameter $\mathrm{E}_{0}$ was chosen according to bremsstrahlung systematics [27, 28]. The contribution was normalized to the experimental spectra at 25-30 MeV and was added to the calculated $\gamma$-ray spectra from CASCADE after folding with detector response. The measured $\gamma$-ray spectra were fitted within the region $\mathrm{E}_{\gamma}=8-25 \mathrm{MeV}$ with CASCADE using a $\chi^{2}$ 

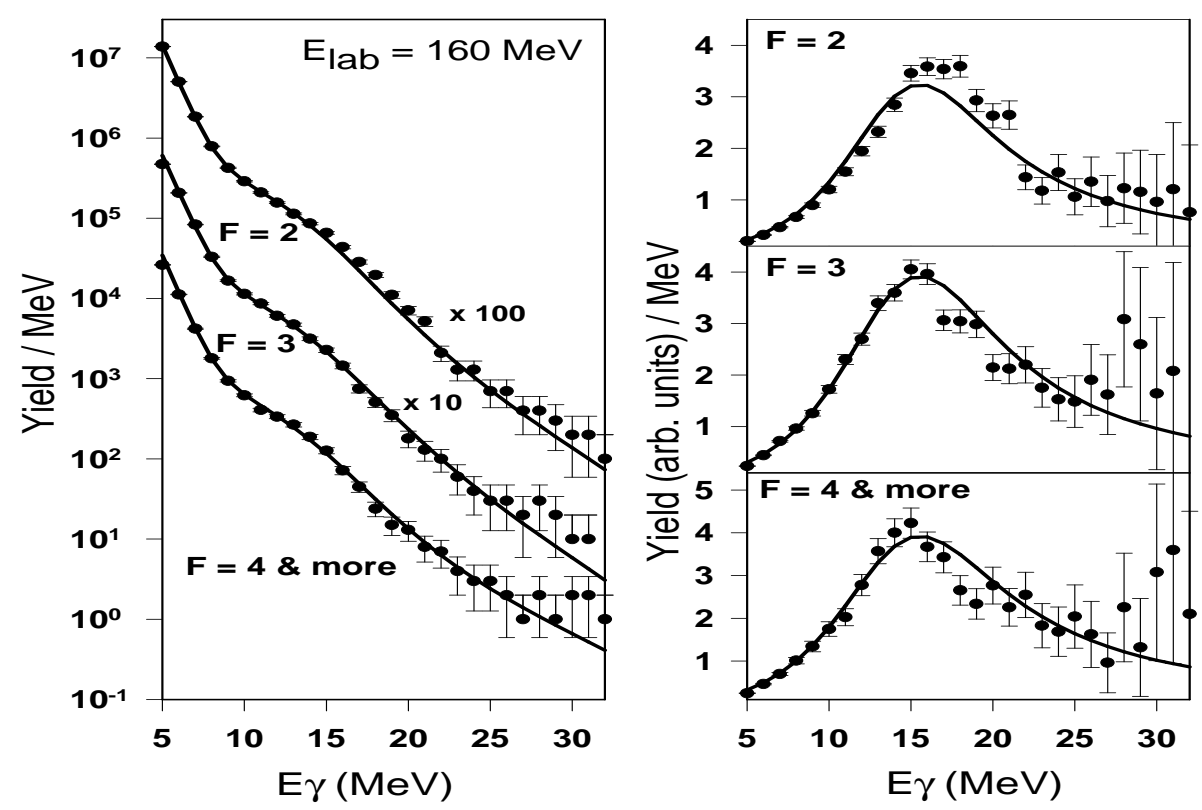

FIG. 7: Left: high energy $\gamma$-ray spectra for different folds at beam energy $160 \mathrm{MeV}$, right: the linearized GDR spectra for different folds plotted against $\gamma$-ray energies.

minimisation routine. The experimental spectra fitted with CASCADE plus bremsstrahlung for different folds are shown in figures 6 and 7 for $\mathrm{E}_{\text {beam }}=145$ and $160 \mathrm{MeV}$ respectively . The linearized GDR plots were extracted by the transformation $f\left(E_{\gamma}\right) * Y_{\gamma}^{e x p} / Y_{\gamma}^{\text {cal }}$ for $\mathrm{E}_{\text {beam }}=145$ and $160 \mathrm{MeV}$ and shown in figures 6 and 7 respectively, where, $Y_{\gamma}^{e x p}$ is the experimental spectrum and $Y_{\gamma}^{c a l}$ is the prediction from CASCADE folded with the detector response. $\mathrm{f}\left(\mathrm{E}_{\gamma}\right)$ is the GDR strength function and is given by,

$$
f\left(E_{\gamma}\right)=\frac{E_{\gamma} \Gamma_{G D R}}{\left[\left(E_{\gamma}^{2}-E_{G D R}^{2}\right)^{2}+E_{\gamma}^{2} \Gamma_{G D R}^{2}\right]}
$$

The values of GDR parameters are shown in Table IV.

\section{Temperature Estimation}

At high excitation energy the compound nucleus decays through a large number of decaying steps, and hence the mass (A), charge $(\mathrm{Z})$, excitation energy $\left(\mathrm{E}^{*}\right)$ and angular momentum $(\mathrm{J})$ of the compound nucleus should be averaged over all the decay steps. The average values of $\mathrm{E}^{*}, \mathrm{~J}, \mathrm{~A}, \mathrm{Z}$ should be different and less than those of the initial compound nucleus. While estimating the average temperature, in accordance with the prescription adopted by 
TABLE IV: Table showing GDR and bremsstrahlung parameters for different beam energies calculated by CASCADE as explained in the text.

\begin{tabular}{|c|c|c|c|c|c|}
\hline $\begin{array}{l}\mathrm{E}_{\text {beam }} \\
(\mathrm{MeV})\end{array}$ & $\begin{array}{c}\text { Fold } \\
(\mathrm{F})\end{array}$ & $\begin{array}{c}\text { Strength } \\
\left(\mathrm{S}_{G D R}\right)\end{array}$ & $\begin{array}{l}\mathrm{E}_{G D R} \\
(\mathrm{MeV})\end{array}$ & $\begin{array}{l}\Gamma_{G D R} \\
(\mathrm{MeV})\end{array}$ & $\begin{array}{c}\mathrm{E}_{0} \\
(\mathrm{MeV})\end{array}$ \\
\hline 145 & 2 & $1.01 \pm 0.01$ & $15.55 \pm 0.10$ & $11.5 \pm 0.25$ & 3.0 \\
\hline 145 & 3 & $0.98 \pm 0.02$ & $15.55 \pm 0.10$ & $11.8 \pm 0.25$ & 3.0 \\
\hline 145 & 4 & $0.99 \pm 0.01$ & $15.55 \pm 0.12$ & $12.4 \pm 0.25$ & 3.0 \\
\hline 145 & $\geq 5$ & $0.98 \pm 0.02$ & $15.55 \pm 0.12$ & $12.8 \pm 0.25$ & 3.0 \\
\hline 160 & 2 & $0.98 \pm 0.02$ & $15.55 \pm 0.12$ & $11.9 \pm 0.25$ & 3.5 \\
\hline 160 & 3 & $0.98 \pm 0.02$ & $15.55 \pm 0.12$ & $12.5 \pm 0.25$ & 3.5 \\
\hline 160 & $\geq 4$ & $0.98 \pm 0.02$ & $15.55 \pm 0.12$ & $13.0 \pm 0.25$ & 3.5 \\
\hline
\end{tabular}

Wieland et al. [29], a lower limit in excitation energy during the CN decay process was employed in the statistical model calculation. This cut in $\mathrm{E}^{*}$ affects the low energy part of the high energy $\gamma$-spectra, without affecting the region of our interest $E_{\gamma}=12-25 \mathrm{MeV}$. The average values of mass, atomic number, pre-equilibrium corrected excitation energy and angular momentum were calculated using the above-mentioned $\mathrm{E}^{*}$ limit. Within this $\mathrm{E}^{*}$ limit, the estimated average values correspond to approximately $50 \%$ of the total high energy $\gamma$-ray yield $\left(E_{\gamma}=12-25 \mathrm{MeV}\right)$ in $\mathrm{CN}$ decay chain. The average temperature was estimated from $\bar{E}^{*}$ by using the relation,

$$
\bar{T}=\left[\left(\bar{E}^{*}-\bar{E}_{\text {rot }}-E_{G D R}-\Delta_{p}\right) / a\left(\bar{E}^{*}\right)\right]^{1 / 2} \text { where } \bar{E}^{*} \text { is the average of the excitation energy }
$$
after pre-equilibrium subtraction weighed over the daughter nuclei for the $\gamma$-emission in the GDR region from $E_{\gamma}=12-25 \mathrm{MeV}$ :

$$
\bar{E}^{*}=\sum_{i}\left(E_{i}^{*} \omega_{i}\right) / \sum_{i} \omega_{i}
$$

$E_{i}^{*}$ is the excitation energy of $\mathrm{i}^{\text {th }}$ nuclei in the decay steps and $\omega_{i}$ is the yield in the region $E_{\gamma}=12-25 \mathrm{MeV} . \mathrm{E}_{\text {rot }}$ is the energy bound in the rotation computed at the average J calculated within the CASCADE corresponding to analysed fold [30, 31]. $\mathrm{E}_{G D R}$ is the centroid energy of the GDR, given by $15.55 \mathrm{MeV}$ and $\Delta_{p}$ is the pairing energy. The details of the parameters for different beam energies calculated by CASCADE for this experiment are shown in Table $\mathrm{V}$. 
TABLE V: Table showing parameters for different beam energies calculated by CASCADE for this experiment.

\begin{tabular}{|c|r|r|r|}
\hline \hline $\mathrm{E}_{\text {beam }}$ & $\mathrm{J}_{C N}$ & $\mathrm{~J}_{\text {mean }}$ & $\mathrm{T}_{\text {mean }}$ \\
$(\mathrm{MeV})$ & $(\hbar)$ & $(\hbar)$ & $1.94_{-0.1}^{+0.06}$ \\
\hline 145 & 49.0 & 41.0 & $1.87_{-0.1}^{+0.06}$ \\
145 & 53.2 & 48.0 & $1.81_{-0.1}^{+0.03}$ \\
145 & 56.7 & 50.0 & $1.72_{-0.03}^{+0.07}$ \\
145 & 59.5 & 54.0 & $1.98_{-0.05}^{+0.14}$ \\
160 & 50.0 & 44.0 & $1.90_{-0.04}^{+0.13}$ \\
160 & 54.3 & 47.0 & $1.86_{-0.14}^{+0.09}$ \\
160
\end{tabular}

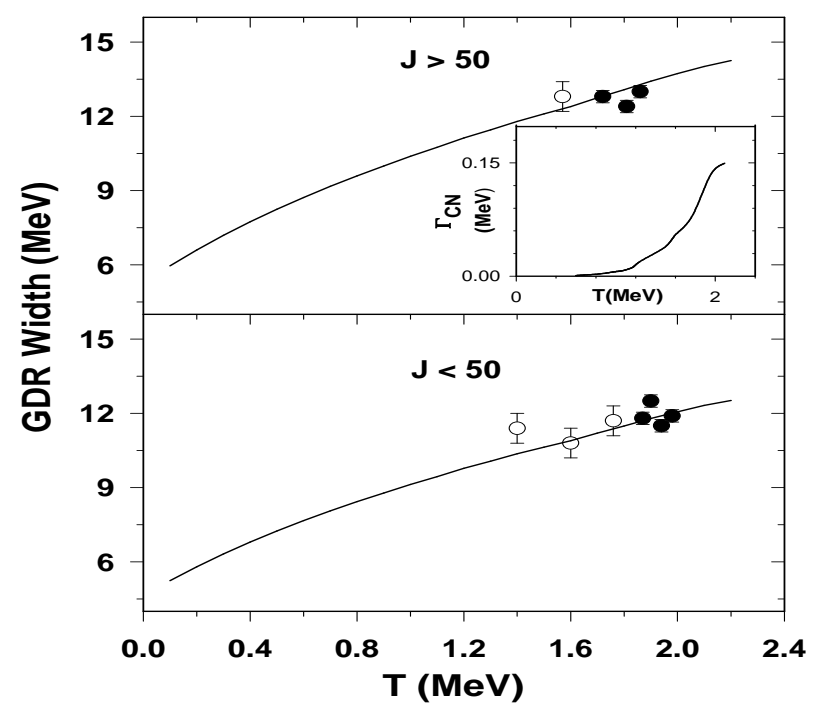

FIG. 8: top: GDR widths are plotted against $\mathrm{T}$ and compared with Kusnezov calculation for $\mathrm{J}>$ $50 \hbar$, bottom: Same plot for $\mathrm{J}<50 \hbar$. The filled circles denote experimental data from this work and the points with the open circles are from Bracco et al [15]. Same scheme for averaging the $\mathrm{T}$ and $\mathrm{J}$ has been adopted in both the cases (see text for details). inset: compound nuclear particle decay width plotted against $\mathrm{T}$.

\section{EXPERIMENTAL RESULTS}

The GDR widths $\Gamma(T, J, A)$ measured in this work are plotted against average values of T. These are shown as filled circles in the top panel of the Fig. 8 for angular momenta 
greater than or equal to $50 \hbar$ and in the bottom panel for angular momenta less than $50 \hbar$. The TSFM predictions using Kusnezov parametrization for $\mathrm{J}>50 \hbar$ and $\mathrm{J}<50 \hbar$ are also represented by solid lines in the top and the bottom panels. The average mass in this case is 111. TSFM calculation (indicated by solid line) includes the average compound nucleus particle decay width calculated with an asymptotic level density parameter $\tilde{a}=\mathrm{A} / 8.0 \mathrm{MeV}^{-1}$ - The graph in the inset of the Fig. 8 shows the average compound nucleus particle decay width plotted against temperature. In this $\mathrm{E}^{*}$ range, the $\Gamma_{C N}$ has small magnitude and grows with increase in $\mathrm{T}$. The inclusion of $\Gamma_{C N}$ improves the fitting in Fig 8 marginally.

There exist an earlier measurement of the GDR width from ${ }^{109,110} \mathrm{Sn}$ at similar temperature and angular momentum by Bracco et al. [15]. A reasonable agreement between those sets of data and predictions by Kusnezov parametrization has been shown in [3]. But recently by employing a new scheme of analysis a large mismatch between the data and the prediction has been seen [18]. The average values of $\mathrm{T}$ and $\mathrm{J}$ have been estimated for this data set also following the approach adopted in this work and is compared with the corresponding TSFM predictions. The results are shown in Figs. 8, 9 \& 10 as open circles (see TableVI for details of the parameters). While the temperature dependence is well-described by the TSFM calculation, the dependence of GDR width on $\mathrm{J}$ is under-predicted as shown in the Fig. 9 (for some points it is more than the error bar).

In Tables $\mathrm{V}$ and $\mathrm{VI}$ the errors in $\mathrm{T}$ indicate the average temperature ranges associated to $80 \%$ (lower value) and $20 \%$ (upper value) of the total high energy $\gamma$-yield in CN decay chain.

The averaging scheme followed in this work, considers only the high energy $\gamma$-rays which really influence the GDR width in the $\mathrm{CN}$ decay chain and neglects the remaining $\gamma$-decay cascade. In this low $\mathrm{E}^{*}$ region, the average values of $\mathrm{T}$ do not differ too much with the initial CN values primarily due to the emission of GDR $\gamma$-rays in the first few steps of the $\mathrm{CN}$ decay cascade. As GDR decay is an average process, the averaging of $\mathrm{E}^{*}, \mathrm{~T}, \mathrm{~A}$ and $\mathrm{J}$ is important in the $\mathrm{CN}$ decay chain. The change of the value of A after averaging from that of the $\mathrm{CN}$ is not expected to make a significant change in the conclusion. But the same is not true for J. Even a change in J by a few units, by the averaging, can indeed alter the reduced width measurements.

The GDR widths measured in this work are also plotted against the average values of J's along with the theoretical predictions for $\mathrm{T}=1.85 \mathrm{MeV}$ and $2.0 \mathrm{MeV}$ (solid and dashed 


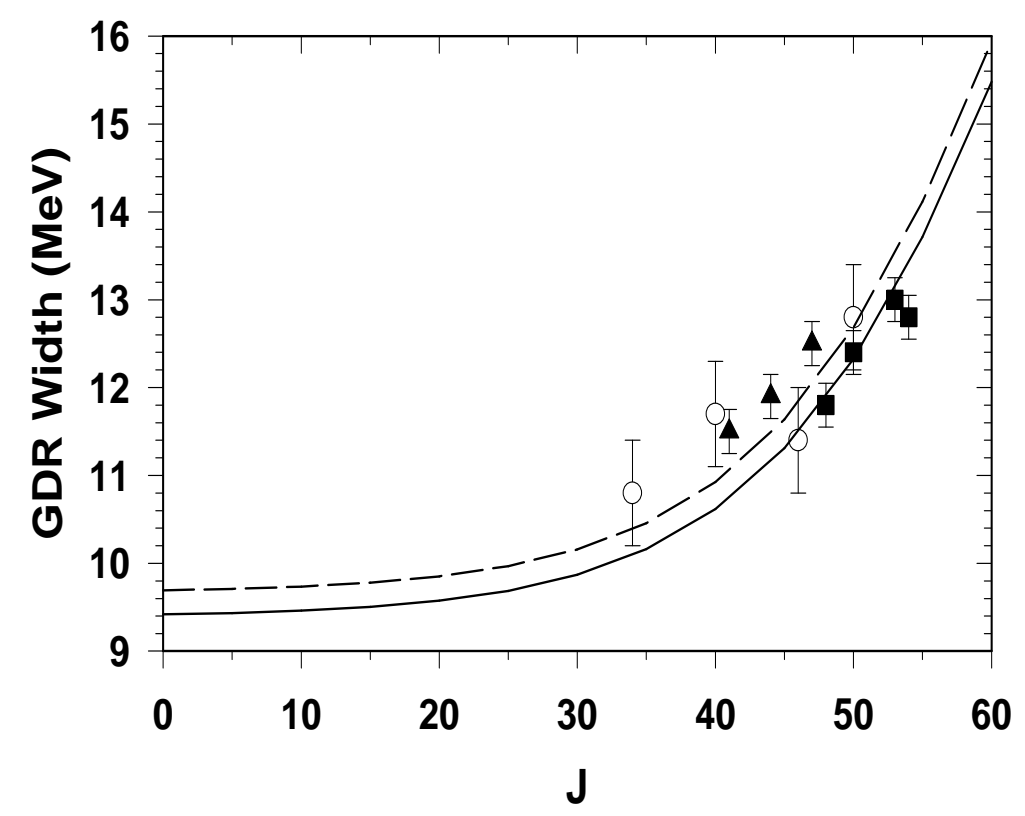

FIG. 9: GDR widths are plotted against $\mathrm{J}$ and compared with Kusnezov calculation for $\mathrm{T}=1.85$ $\mathrm{MeV}$ (solid line) and $2.0 \mathrm{MeV}$ (dashed line). The filled squares and triangles are data from this work while the points with the open circles are from experiment done by Bracco et al. [15] (Details are described in the text).

TABLE VI: Table showing recalculation of parameters in this paper for different beam energies calculated by CASCADE for the experiment performed by Bracco et al.(see [15])

\begin{tabular}{|c|c|c|c|c|c|c|}
\hline $\begin{array}{l}\mathrm{E}_{\text {beam }} \\
(\mathrm{MeV})\end{array}$ & $\begin{array}{c}\mathrm{J}_{C N} \\
(\hbar)\end{array}$ & $\begin{array}{c}\mathrm{J}_{\text {mean }} \\
(\hbar) \\
\end{array}$ & $\begin{array}{l}\mathrm{T}_{\text {mean }} \\
(\mathrm{MeV})\end{array}$ & $\begin{array}{l}\mathrm{E}_{G D R} \\
(\mathrm{MeV}) \\
\end{array}$ & $\begin{array}{l}\Gamma_{G D R} \\
(\mathrm{MeV}) \\
\end{array}$ & $\begin{array}{c}\text { FWHM in } \mathrm{J} \\
(\hbar)\end{array}$ \\
\hline 223 & 44.0 & 40.0 & $1.76_{-0.02}^{+0.15}$ & $15.0 \pm 0.5$ & $11.7 \pm 0.6$ & 16 \\
\hline 223 & 54.0 & 50.0 & $1.57_{-0.01}^{+0.12}$ & $14.7 \pm 0.5$ & $12.8 \pm 0.6$ & 14 \\
\hline 203 & 40.0 & 34.0 & $1.60_{-0.05}^{+0.13}$ & $15.7 \pm 0.5$ & $10.8 \pm 0.6$ & 18 \\
\hline 203 & 49.0 & 46.0 & $1.40_{-0.03}^{+0.08}$ & $15.6 \pm 0.5$ & $11.4 \pm 0.6$ & 16 \\
\hline
\end{tabular}

lines respectively in the Fig. 9). The data points represented by solid squares and triangles are for $\mathrm{T} \leq 1.85 \mathrm{MeV}$ and $1.85<\mathrm{T}<2.0 \mathrm{MeV}$ respectively. They are agreeing well with Kusnezov parametrization. If the CN decay widths are considered (not shown in the figure), the matching improves marginally.

In the Fig. 10, reduced GDR widths are plotted for this experiment as well as for the other set of existing data. The solid line corresponds to the global phenomenological free energy surface calculation shown in [12] at $\mathrm{T}=1.8 \mathrm{MeV}$ within the TSFM. Reduced GDR widths $\Gamma_{\text {red }}$ for this experiment are shown in the figure by filled circles and the data for 


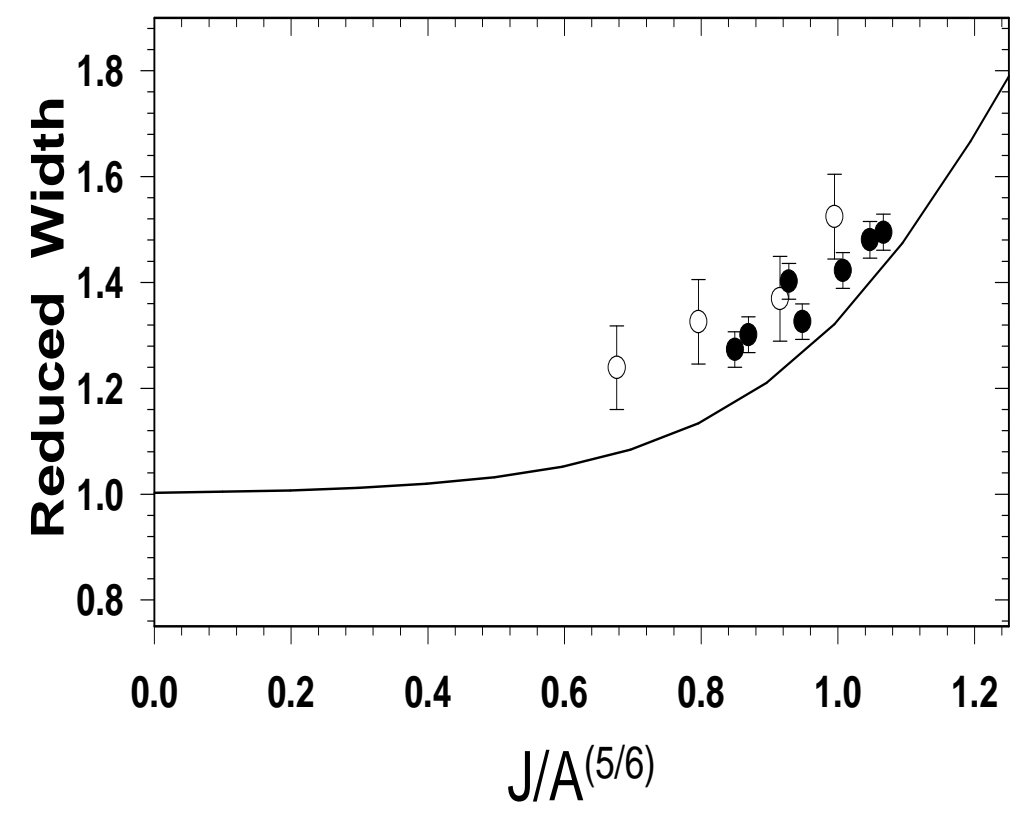

FIG. 10: Reduced GDR widths are plotted against $\xi=J / A^{5 / 6}$ and compared with Kusnezov calculation. The filled circles are data from this work while the points with the open circles are from experiment done by Bracco et al. [15]

Bracco et al. are shown by open circles. Those reduced GDR widths are calculated using the parametrization,

$$
\Gamma_{\text {red }}=\Gamma_{e x p}(T, J, A) / \Gamma_{\text {theory }}(T, J=0, A)^{\left(T+3 T_{0}\right) /\left(4 T_{0}\right)}
$$

and plotted against $\xi=\bar{J} / \bar{A}^{5 / 6}$, where $\mathrm{T}_{0}=1 \mathrm{MeV}$ and ground state GDR width $\Gamma_{0}=3.8$ $\mathrm{MeV}$. Although the simple parametrization proposed by Kusnezov reproduces reasonably well the trend of the experimental findings as a function of spin, it would be interesting to see whether the more detailed calculation with the Thermal Shape Fluctuation Model for specific nuclei can improve further the agreement with the data in reduced width plot for higher spins. 


\section{SUMMARY AND CONCLUSION}

The GDR width built on excited states of ${ }^{113} \mathrm{Sb}$ has been studied in the interval $\mathrm{J}=$ $40-60 \hbar$ and at a temperature ranging from $\mathrm{T}=1.7-2.0 \mathrm{MeV}$ using a part of the LAMBDA array. To decouple the effect of $\mathrm{T}$ and $\mathrm{J}$ from each other on GDR width, multiplicity detector assembly has been used. The angular momentum information was obtained from the low energy $\gamma$-multiplicity filter. Pre-equilibrium corrections on the excitation energies were performed. The temperature has been found from the average values of corrected excitation energy and angular momentum which provided a more stringent test for the existing TSFM for explaining the systematics of GDR width. Another set of existing data from earlier measurement of GDR width of Sn nuclei have been used for comparison. In spite of the fact that the simple parametrization of Kusnezov describes the trend well, there remains a scope of detail TSFM calculation for further improvement in the parametrization of GDR width with J. Keeping $\mathrm{J}$ constant, the increase of $\Gamma$ with $\mathrm{T}$, for $\mathrm{T} \leq 2.0 \mathrm{MeV}$, is well understood by TSFM although the matching improves to some extent by the addition of CN decay width. So for a complete understanding of thermal shape fluctuation and parametrization of GDR width, more experiments in this mass range are needed. Also in the higher temperature region it is important to find out the detail pre-equilibrium energy loss involved.

The authors wish to thank I.Dioszegi and D.Hoffman for their help and suggestion regarding the modification of CASCADE.

* e-mail:srb@veccal.ernet.in

[1] J.J.Gaardhoje, Annu. Rev. Nucl. Part. Sci, 42, 483 (1992).

[2] K.Snover, Annu. Rev. Nucl. Part. Sci, 36, 545 (1986).

[3] M.Thoennessen, Nucl. Phys. A731, 131 (2004).

[4] V.Baran et al., Nucl. Phys. A599, 29c (1996).

[5] W.E.Ormand et al., Nucl. Phys. A614, 217 (1997).

[6] W.E.Ormand et al., Phys. Rev. Lett, 77, 607 (1996).

[7] P.F.Bortignon et al., Giant Resonances Nuclear Structure at Finite Temperature, Harwood Academic Pub. 
[8] A.Bracco et al., Phys. Rev. Lett, 62, 2080 (1989).

[9] H.J.Hofman et al., Nucl. Phys. A571, 301 (1994).

[10] D.Pierroutsakou et al., Nucl. Phys. A600, 131 (1996).

[11] M.P.Kelly et al., Nucl. Phys. A649, 123c (1999).

[12] D. Kusnezov et al., Phys. Rev. Lett, 81, 542 (1998).

[13] T. Baumann et al., Nucl. Phys. A635, 428 (1998).

[14] P. Heckman et al., Phys. Lett B555, 43 (2003).

[15] A.Bracco et al., Phys. Rev. Lett, 74, 3748 (1995).

[16] M.Matiuzzi et al., Nucl. Phys. A 612, 262 (1997).

[17] S.K.Rathi et al., Phys. Rev. C67, 024603 (2003).

[18] D.R.Chakrabarty et al., Phys. Rev. C74, 017601 (2006).

[19] Srijit Bhattacharya et al., Proc. I.N.P.C, Tokyo, 2007.

[20] S. Mukhopadhyay et al., Nucl. Instr. Meth. A 582, 603 (2007).

[21] R. Brun et al., GEANT3 CERN-DD/EE/84-1, 1986.

[22] Srijit Bhattacharya et al., Proc. DAE-BRNS Symposium, Banaras, 2004.

[23] F.Puhlhoffer et al., Nucl. Phys. A 280, 267 (1967).

[24] H.Tricoire et al., Z. Phys. A 306, 127 (1982).

[25] W.Reisdorf et al., Z. Phys. A 300, 227 (1981).

[26] I.Dioszegi et al., Phys. Rev. C61, 024613 (2000).

[27] H. Nifenecker et al., Annu. Rev. Nucl. Part. Sci, 40, 113 (1990).

[28] Ramakrishnan et al., Phys. Rev. Lett, 76, 2025 (1996).

[29] Wieland et al., Phys. Rev. Lett, 97, 012501 (2006).

[30] M.Kmiecik et al., Nucl. Phys. A674, 29 (2000).

[31] G.Gervais et al., Nucl. Phys. A649, 173c (1999). 